\title{
Evaluation of the Right Atrial Venous Oxygen Saturation as a Physiologic Monitor in a Neonatal Model
}

\author{
By Ronald B. Hirschl, Palle Palmer, Kurt F. Heiss, Karl Hultquist, Franco Fazzalari, and Robert H. Bartlett \\ Ann Arbor, Michigan
}

\begin{abstract}
- Pulmonary artery (PA) mixed venous saturation $\left(\mathrm{Sv}_{2}\right)$ has become a crucial monitor in the adult intensive care unit, but is not used in neonates because of the difficulty in PA catheterization. We evaluated the possibility of utilizing the right atrial venous oxygen saturation $\left(\mathrm{RA} \overline{\mathrm{v}} \mathrm{O}_{2}\right)$, which is easily accessed in the neonate, as a monitor of the effects of mechanical ventilation and intravascular volume in an animal model selected to be the size of the human neonate. A continuous $\mathrm{RAvO}_{2}$ monitoring catheter was placed into the right atrium of 16 normal rabbits $(2.2$ to $4.1 \mathrm{~kg}$ ). Oxygen delivery was manipulated by alterations in peak inspiratory pressure (PIP) ( $n=6)$, positive end-expiratory pressure (PEEP) $(n=6)$, or by progressive hypovolemia $(n=4) . \mathrm{RAvO}_{2}$ decreased with onset of mechanical ventilation alone from $69 \% \pm 6 \%$ to $61 \% \pm 5 \%(P<.01)$. As the PIP was increased from 12 to $21 \mathrm{~cm} \mathrm{H} \mathrm{H}_{2} \mathrm{O}$, the $\mathrm{RAvO}_{2}$ progressively decreased from $59 \% \pm 4 \%$ to $49 \% \pm 6 \%(P<.05)$. As the PEEP was increased from 3 to $9 \mathrm{~cm} \mathrm{H}_{2} \mathrm{O}$, the $\mathrm{RAvO}_{2}$ progressively decreased from $64 \% \pm 5 \%$ to $33 \% \pm 16 \%(P<.01)$. RAVO approached baseline after return to continuous positive airway pressure (CPAP) of $3 \mathrm{~cm} \mathrm{H}_{2} \mathrm{O}$. Progressive phlebotomy to a total of $10 \mathrm{~mL} / \mathrm{kg}$ resulted in a decrease in $\mathrm{RAVO}_{2}$ from $70 \% \pm 6 \%$ to $27 \% \pm 5 \%(P<.001)$. Volume resuscitation resulted in an increase in $\mathrm{RAv}_{2}$ to near baseline. Peripheral arterial oxygen saturation remained at a constant $100 \%$ throughout each protocol. With oxygen consumption stable, the $\mathrm{RAvO}_{2}$ is an excellent monitor of the effect of airway pressure or of hypovolemia on oxygen delivery. Use of the peripheral arterial oxygen saturation alone as a parameter for monitoring ventilator or intravascular volume effect is not adequate. $\mathrm{RAvO}_{2}$ monitoring may be a powerful tool in the management of the critically ill neonate.

Copyright 1993 by W.B. Saunders Company
\end{abstract}

INDEX WORDS: Right atrial venous oxygen saturation $\left(\mathrm{RA}_{\bar{v}} \mathrm{O}_{2}\right.$ ) monitoring, neonate.

$\mathbf{M}$ OST NEWBORNS with respiratory failure are treated effectively with mechanical ventilation. ${ }^{1-3}$ Evaluation of the overall physiologic effect of mechanical ventilation, however, is hindered in this age group becausc of an inability to cvaluate the cardiac output and oxygen consumption. Positivepressure ventilation (PPV) and positive end-expiratory pressure (PEEP) are, therefore, applied in neonates without a means for evaluating their influence on oxygen delivery. In children and adults, both the cardiac output and the oxygen consumption may be routinely monitored and the oxygen delivery optimized in relation to oxygen consumption. In these patients, the pulmonary artery mixed venous oxygen saturation $\left(\mathrm{S}_{\bar{v}} \mathrm{O}_{2}\right)$ is a reliable and effective method of monitoring the relationship between oxygen delivery and oxygen consumption. As long as oxygen consump- tion remains relatively constant, the $S \bar{v} O_{2}$ is used to titrate the effect of PEEP and PPV on oxygen delivery. Pulmonary artery measurement of venous saturation for these purposes is considered essential because of the variability of venous saturation in the superior vena cava, the inferior vena cava, and the coronary sinus, along with inadequate mixing in the right atrium. However, since bedside access to the pulmonary artery is difficult in neonates and since mixing in the small right atrium of the newborn might be better than in the adult, we considered whether the right atrial venous oxygen saturation $\left(\mathrm{RA}_{\bar{v}} \mathrm{O}_{2}\right)$ might provide information similar to that of the $\mathrm{S}_{\bar{v}} \mathrm{O}_{2}$. Therefore, the purpose of this study was to evaluate the effectiveness of the right atrial venous oxygen saturation as a monitor of the influence of PPV, PEEP, and intravascular volume on oxygen delivery in a neonatal-sized rabbit model.

\section{MATERIALS AND METHODS}

\section{Animal Preparation}

Sixteen normal rabbits 2.2 to $4.1 \mathrm{~kg}$ in weight (mean, $2.9 \mathrm{~kg}$ ) were anesthetized with ketamine, $40 \mathrm{mg} / \mathrm{kg}$. Subsequent surgical levels of anesthesia were maintained by administration of a $10 \mathrm{mg} / \mathrm{kg} / \mathrm{h}$ ketamine infusion. No paralyzing agents were used. Tracheostomy was performed with placement of a 3.5- to 4.0-mm inner diameter cuffed tracheostomy tube. A 5F catheter was introduced into the right carotid artery and used for blood pressure monitoring as well as for arterial blood gas analysis. A 4F Oximetrix (Mountainview, CA) fiber-optic venous oxygen saturation catheter was placed into the right internal jugular vein and advanced until right ventricular pressures and waveforms were noted on monitor. A right ventricular blood sample was drawn. The catheter was withdrawn until right atrial pressures and waveforms were observed. A right atrial venous sample was analyzed and in vivo calibration of the fiberoptic catheter performed. Location of the catheter tip was documented by autopsy at conclusion of the protocol.

\section{Mechanical Ventilation Protocol}

Animals were first allowed to breathe spontaneously on a continuous positive airway pressure (CPAP) of $3 \mathrm{~cm} \mathrm{H} \mathrm{H}_{2} \mathrm{O}$. They were then mechanically ventilated by one of two protocols: (1) in 6 rabbits the PIP was progressively increased from 12 to $21 \mathrm{~cm} \mathrm{H}_{2} \mathrm{O}$

From the Department of Surgery, University of Michigan, Ann Arbor, MI

Date accepted: July 14, 1992.

Address reprint requests to Ronald B. Hirschl, MD, L2110 Maternal and Child Health Center, $1500 \mathrm{E}$ Medical Center Dr, University of Michigan Hospitals, Ann Arbor, MI 48109-0245.

Copyright 01993 by W.B. Saunders Company 0022-3468/93/2807-0008\$03.00/0 
in increments of $3 \mathrm{~cm} \mathrm{H} \mathrm{H}_{2} \mathrm{O}$ while the PEEP was held constant at 3 $\mathrm{cm} \mathrm{H}_{2} \mathrm{O}$; and (2) in 6 rabbits the PEEP was increased from 3 to 9 $\mathrm{cm} \mathrm{H}_{2} \mathrm{O}$ in increments of $2 \mathrm{~cm} \mathrm{H} \mathrm{H}_{2} \mathrm{O}$ while the PIP was held constant at $15 \mathrm{~cm} \mathrm{H} \mathrm{H}_{2} \mathrm{O}$.

All animals were then removed from mechanical ventilation and

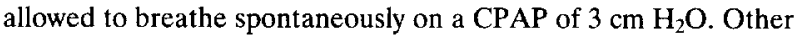
ventilatory parameters were held constant during periods of mechanical ventilation with a respiratory rate of $30 \mathrm{breaths} / \mathrm{min}$, $\mathrm{FiO}_{2} 40 \%$, inspiratory time 0.5 seconds, and flow rate of $6 \mathrm{~L} / \mathrm{min}$. Mechanical ventilation was performed utilizing a Sechrist IV100-B ventilator (Sechrist Industries, Anaheim, CA) and airway pressures, including the mean, peak, and end-expiratory pressures were measured at the level of the tracheostomy tube via a Sechrist mean airway pressure monitor.

\section{Hypovolemia Protocol}

Four rabbits were mechanically ventilated at constant ventilator settings with PIP $=13$, PEEP $=4$, respiratory rate of 30 breaths $/ \mathrm{min}, \mathrm{FiO}_{2} 40 \%$, inspiratory time 0.5 seconds, and flow rate of $6 \mathrm{~L} / \mathrm{min}$. Sequential withdrawal of $2 \mathrm{~mL} / \mathrm{kg}$ of whole blood from the right atrial venous catheter was performed every 10 minutes until a total of $10 \mathrm{~mL} / \mathrm{kg}$ had been evacuated into a heparinized syringe. Volume repletion of $10 \mathrm{~mL} / \mathrm{kg}$ of crystalloid solution was then performed after an additional 10-minute waiting period.

\section{Oxygen Saturation Measurements}

Venous blood was analyzed for oxygen saturation independently by continuous venous fiber-optic oximetry and by an IL282 cooximeter. Arterial blood samples underwent IL282 co-oximeter analysis for hemoglobin and oxygen saturation, as well as blood gas evaluation by an ABL blood gas analyzer (Radiometer, Copenhagen, Denmark).

\section{Data Analysis}

At each point of CPAP, after each change in mechanical ventilation, and after each change in intravascular volume the animal was allowed to stabilize over a 10-minute period. Physiologic data such as heart rate, respiratory rate, and blood pressure were then obtained. Blood was drawn for venous oxygen saturation analysis and arterial blood gas, hemoglobin, and oxygen saturation analysis. The Oximetrix catheter venous oxygen saturation was recorded. All data were averaged for each point of CPAP or mechanical ventilation and statistically analyzed by paired Student's $t$ test. All animals were cared for under the standards and practices of the University of Michigan Laboratory Animal Care facilities.

\section{RESULTS}

Comparison of the measurements of right ventricular and right atrial venous oxygen saturation is evaluated in Fig 1. Correlation was excellent $(r=.98)$. The mean right atrial venous oxygen saturation was $61 \%$ and the mean right ventricular oxygen saturation was $59 \%$. There was no significant difference between these values.

Venous and arterial oxygen saturation data during periods of CPAP and mechanical ventilation are presented in Figs 2 and 3. While spontaneously breathing on CPAP of $3 \mathrm{~cm} \mathrm{H}_{2} \mathrm{O}$, the mean $\mathrm{RAv}_{2}$ in all 12 animals was $69 \% \pm 6 \%$. Onset of mechanical ventilation alone resulted in a significant decrease in mean $\mathrm{RA} \bar{v} \mathrm{O}_{2}$ to $61 \% \pm 5 \%(P<.01)$.

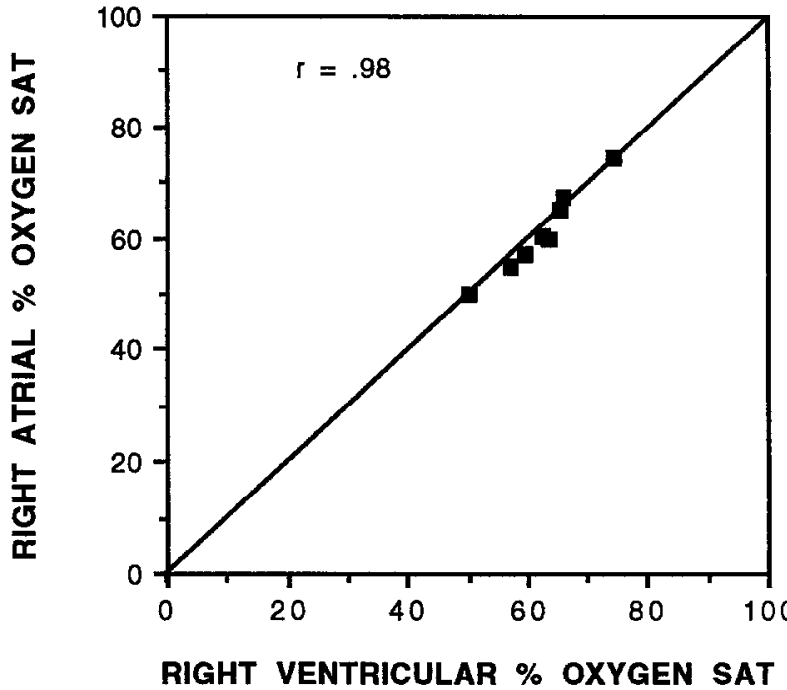

Fig 1. Comparison of right atrial and right ventricular \% oxygen saturation measured by co-oximetry. Correlation coefficient $r=.98$.

As the PIP was sequentially increased from 12 to 21 with the PEEP held constant, the $\mathrm{RA} \overline{\mathrm{v}} \mathrm{O}_{2}$ decreased progressively from $59 \% \pm 4 \%$ to $49 \% \pm 6 \%(P<.05)$. The measured mean airway pressure increased simultaneously from $3.7 \pm 0.9$ to $4.8 \pm 1.1 \mathrm{~cm} \mathrm{H}_{2} \mathrm{O}$. Removal from mechanical ventilation and resumption of spontaneous breathing on CPAP of $3 \mathrm{~cm} \mathrm{H}_{2} \mathrm{O}$ resulted in a significant increase of the $\mathrm{RAv}_{2} \mathrm{O}_{2}$ to $63 \% \pm 8 \%(P<.01)$. The arterial oxygen saturation $\left(\mathrm{SaO}_{2}\right)$ remained at $100 \%$ throughout.

As the PEEP was increased sequentially from 3 to 9 cm $\mathrm{H}_{2} \mathrm{O}$ with the PIP held constant, the $\mathrm{RA} \bar{v} \mathrm{O}_{2}$ progressively decreased from $64 \% \pm 5 \%$ to $33 \% \pm$ $16 \%(P<.01)$. The measured mean airway pressure increased simultaneously from $4.3 \pm 0.7$ to $9.6 \pm 0.5$ $\mathrm{cm} \mathrm{H}_{2} \mathrm{O}$. Removal of these animals from mechanical

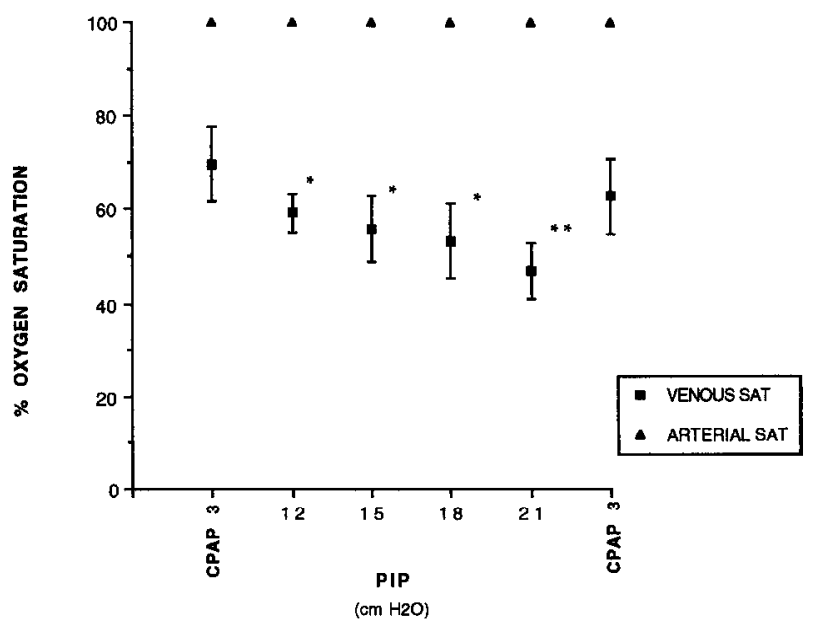

Fig 2. Venous and arterial $\%$ oxygen saturation while on CPAP $=3$ $\mathrm{cm} \mathrm{H}_{2} \mathrm{O}$ and with sequential increase in PIP from 12 to $21 \mathrm{~cm} \mathrm{H}_{2} \mathrm{O}$. *P< .05 from baseline; ${ }^{* *} P<.01$ from baseline. 


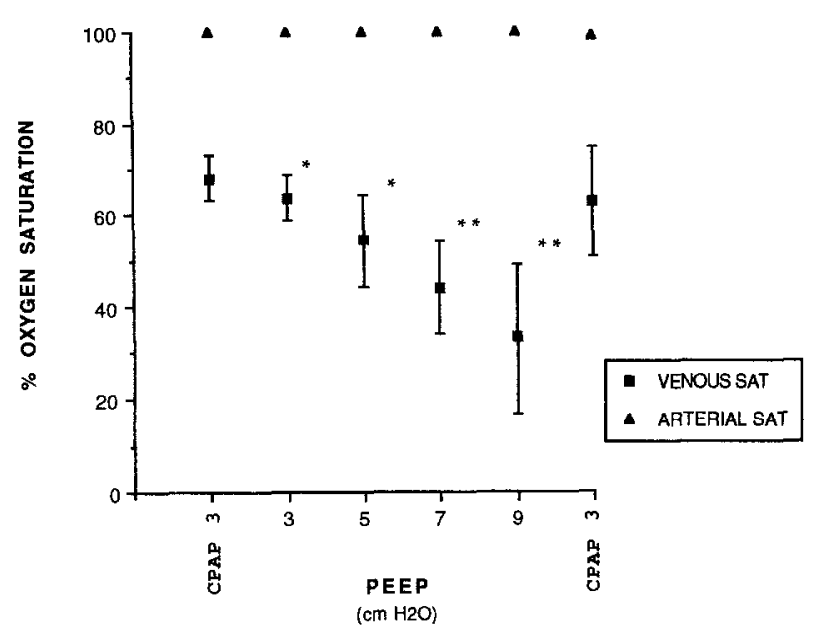

Fig 3. Venous and arterial $\%$ oxygen saturation while on CPAP $=3$ $\mathrm{cm} \mathrm{H}_{2} \mathrm{O}$ and with sequential increase in PEEP from 3 to $9 \mathrm{~cm} \mathrm{H}_{2} \mathrm{O}$. ${ }^{*} P<$ 05 from baseline; ${ }^{*} P<.01$ from baseline.

ventilation resulted in a significant increase in $\mathrm{RA} \bar{v} \mathrm{O}_{2}$ to $63 \% \pm 12 \%(P<.601)$. Peripheral arterial oxygen saturation once again remained at $100 \%$.

The effect of sequential phlebotomy of $2 \mathrm{~mL} / \mathrm{kg}$ at a time to a total of $10 \mathrm{~mL} / \mathrm{kg}$ is demonstrated in Fig 4 . A decrease in the $\mathrm{RA} \bar{v} \mathrm{O}_{2}$ from an initial $70 \% \pm 6 \%$ to $27 \% \pm 5 \%(P<.001)$ was observed. The $\mathrm{RA} \bar{v} \mathrm{O}_{2}$ increased significantly to $65 \% \pm 3 \%(P<.001)$ upon repletion of volume utilizing the evacuated blood. Peripheral arterial oxygen saturation was $100 \%$ throughout.

Correlation of independent analysis of the $\mathrm{RA} \overline{\mathrm{v}} \mathrm{O}_{2}$ by the Oximetrix fiberoptic catheter and the IL282 co-oximeter is presented in Fig 5. The fiberoptic catheter values correlated well with the co-oximeter results $(r=.92)$.

\section{DISCUSSION}

Since the 1960s the effects of mean airway pressure during PPV and PEEP on cardiac output have been

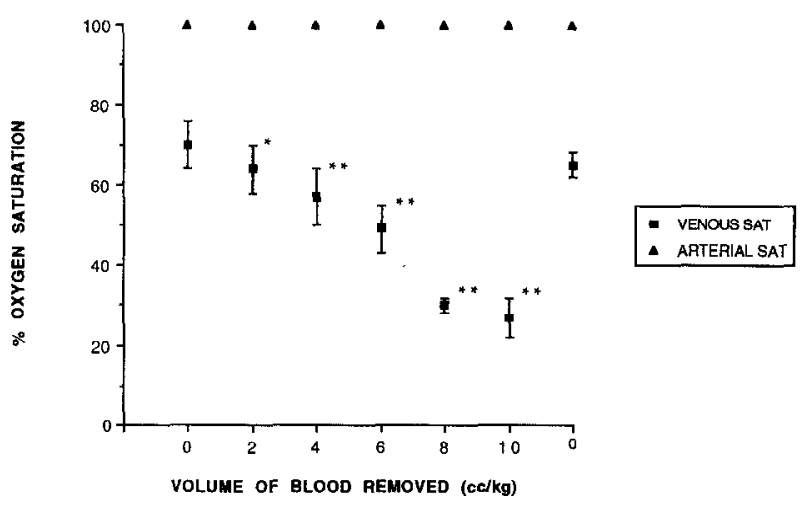

Fig 4. Venous and arterial \% oxygen saturation with induced hypovolemia by sequential removal of whole blood followed by replacement of evacuated volume. ${ }^{*} P<.05$ from baseline; ${ }^{* *} P<.001$ from baseline.

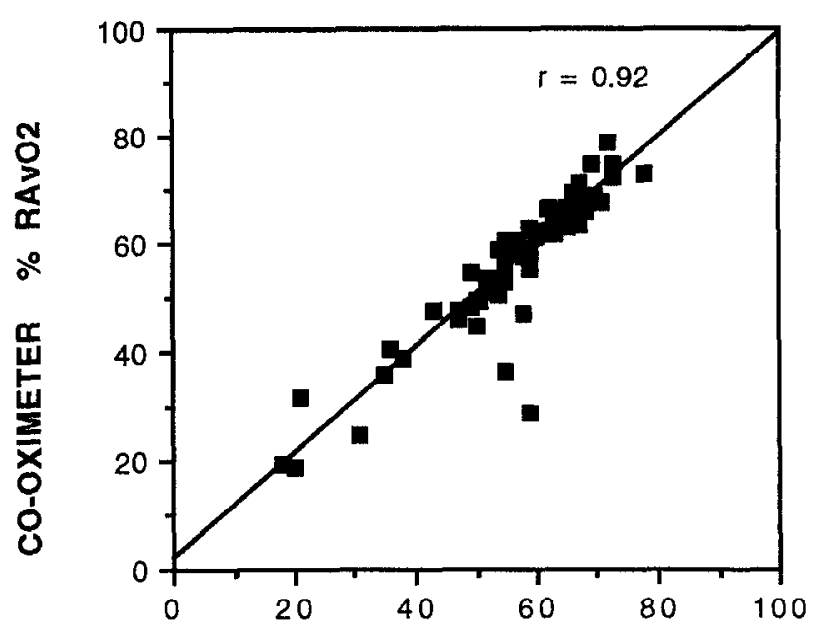

CATHETER OXIMETER \% RAVO2

Fig 5. Comparison of right atrial $\%$ venous oxygen saturation as measured by IL282 co-oximetry or by Oximetrix fiberoptic catheter oximetry. Correlation coefficient $r=.92$.

studied extensively in the animal and adult human population. 4.5 Decrease in cardiac output and left ventricular end-diastolic diameter as well as increase in right atrial pressure, inferior vena caval dimensions, and right ventricular end-diastolic diameter have been documented with increasing levels of PEEP. ${ }^{6-8}$ This may be caused by reduction in venous return, leftward displacement of the intraventricular septum, or reflex depression of myocardial function. ${ }^{9-15}$ The deterioration in hemodynamics induced by application of PEEP is reversed by volume administration. ${ }^{16,17}$ This would tend to suggest that the effects of PEEP on cardiac output are secondary to changes in volume and left ventricular preload. ${ }^{18}$

In the neonatal population, Gabriele et al demonstrated a $20 \%$ reduction in cardiac output in spontaneously breathing newborn lambs after application of CPAP of $11 \mathrm{~cm} \mathrm{H}_{2} \mathrm{O} \cdot{ }^{19}$ Maayan et al demonstrated that left ventricular (LV) dimensions, LV shortening fraction, LV stroke volume, and cardiac output decreased significantly with onset of mechanical ventilation with a mean PEEP of $3.5 \mathrm{~cm} \mathrm{H}_{2} \mathrm{O}$ and a mean peak inspiratory pressure of $20 \mathrm{~cm} \mathrm{H}_{2} \mathrm{O}$ in premature newborns with respiratory distress syndrome. ${ }^{20} \mathrm{Vol}$ ume loading before onset of mechanical ventilation prevented the detrimental hemodynamic effects of PPV. In a similar group of patients, Hausdorf and Hellwege reported significant impairment in right ventricular and LV stroke volume with progressive increase in PEEP from 0 to $8 \mathrm{~cm} \mathrm{H}_{2} \mathrm{O}$. ${ }^{21}$ Despite these findings, normal systemic blood pressure and arterial oxygen saturation were maintained and these patients demonstrated no observable clinical evidence 
of deterioration of cardiac output or oxygen delivery with onset of mechanical ventilation.

In the anesthetized animal or critically ill neonate, the metabolic rate, measured as oxygen consumption, remains relatively stable for hours at a time. ${ }^{22}$ Therefore, changes in oxygen delivery are reflected immediately as changes in venous oxygen content, measured as the $\mathrm{S}_{\bar{v}} \mathrm{O}_{2}$. Increasing airway pressures might increase delivery by increasing arterial oxygen saturation or decrease oxygen delivery by decreasing cardiac output. ${ }^{23}$ Additionally, intravascular volume changes may alter the effect of ventilator pressure manipulations. One way to evaluate these effects is to measure cardiac output and arterial content at each incremental level of airway pressure. ${ }^{24}$ However, this is time-consuming in adults and impractical in infants because of difficulty in measuring cardiac output. A better way to evaluate the effect of airway pressure and intravascular volume manipulation is to measure the $\mathrm{Sv}_{2}{ }^{25}$ This is standard practice in the adult intensive care unit, but cannot be accomplished in neonates because pulmonary artery catheterization is not routincly practical. Numerous studics have previously demonstrated excellent correlation between the central venous blood oxygen saturation and the mixed venous oxygen saturation in animals during various levels of hypoxia, hemorrhage, and resuscitation..$^{26-29}$ Although the limitations of this closed-chest model precluded pulmonary arterial access, our findings document that the values of the $\mathrm{RA} \overline{\mathrm{v}} \mathrm{O}_{2}$, which can be measured continuously, correlate well with the effect expected from elevated airway pressures, as well as decreased cardiac output secondary to induced hypovolemia in rabbits the size of human neonates.

The $\mathrm{RA} \overrightarrow{\mathrm{v}} \mathrm{O}_{2}$ appears to provide excellent monitoring of the effect of PPV, PEEP, and intravascular volume on overall oxygen delivery in normal rabbits. Use of the $\mathrm{SaO}_{2}$ alone as a parameter for evaluating the effect of airway pressures or intravascular volume status on oxygen delivery does not appear to be adequate. Utilization of the $\mathrm{RA} \overline{\mathrm{v}} \mathrm{O}_{2}$ as a monitor has yet to be investigated in neonates. One concern that is present is the potential for left-to-right blood shunting through a patent foramen ovale. Although rightto-left blood shunting predominates in newborns with PPHN, bidirectional shunts through the foramen ovale have been documented. The presence of a left-to-right atrial shunt might elevate the $\mathrm{R} \Lambda \bar{v} \mathrm{O}_{2}$ and introduce error into the interpretation of the absolute $\mathrm{RA} \overline{\mathrm{v}} \mathrm{O}_{2}$ value. Whether this is an appropriate concern can only be determined through clinical evaluation. Based on the data from this study, however, the $\mathrm{RA} \overline{\mathrm{v}} \mathrm{O}_{2}$ appears to have the potential for being a powerful tool for the monitoring of effective oxygenation in the critically ill neonate.

\section{REFERENCES}

1. Delivoria-Papadopoulos M, Levison H, Swyer PR: Intermittent positive pressure respiration as a treatment in severe respiratory distress syndrome. Arch Dis Child 40:474-479, 1965

2. Thomas DV, Fletcher G, Sunshine P, et al: Prolonged respirator use in pulmonary insufficiency of newborn. JAMA 193:183-190, 1965

3. Gregory GA, Kitterman JA, Phibbs RH, et al: Treatment of the idiopathic respiratory-distress syndrome with continuous positive airway pressure. N Engl J Med 284:1331-1340, 1971

4. Kilburn KH, Sieker HO: Hemodynamic effects of continuous positive and negative pressure breathing in normal man. Circ Res 8:660-667, 1960

5. Kumar A, Falke FJ, Geffin B, et al: Continuous positivepressure ventilation in acute respiratory failure. $\mathrm{N}$ Engl $\mathrm{J}$ Med 283:1430-1436, 1970

6. Mitaka C, Nagura T, Sakanishi N, et al: Two-dimensional echocardiographic evaluation of inferior vena cava, right ventricle, and left ventricle during positive-pressure ventilation with varying levels of positive end-expiratory pressure. Crit Care Med 17:205210, 1989

7. Prewitt RM, Wood LDH: Effect of positive end-expiratory pressure on ventricular function in dogs. Am J Physiol 236:H534H544, 1979

8. Cassidy SS, Eschenbacher WL, Robertson CH, et al: Cardiovascular effects of positive-pressure ventilation in normal subjects. J Appl Physiol Respirat Environ Exercise Physiol 47:453-461, 1979

9. Marini JJ, Culver BH, Butler J: Effect of positive endexpiratory pressure on canine ventricular function curves. J Appl Physiol Respirat Environ Exercise Physiol 15:1367-1374, 1981
10. Rankin JS, Olsen GO, Arentzen CE, et al: The effects of airway pressure on cardiac function in intact dogs and man. Circulation 66:108-120, 1982

11. Calvin JE, Driedger AA, Sibhald WI: Positive endexpiratory pressure (PEEP) does not depress left ventricular function in patients with pulmonary edema. Am Rev Respir Dis 124:121-128, 1981

12. Viquerat CE, Righetti A, Suter PM: Biventricular volumes and function in patients with adult respiratory distress syndrome ventilated with PEEP. Chest 83:509-514, 1983

13. Jardin F, Farcot JC, Boisant L, et al: Influence of positive end-expiratory pressure on left ventricular performance. N Engl J Med 304:387-392, 1981

14. Cassidy SS, Robertson CH, Pierce AK, et al: Cardiovascular effects of positive end-expiratory pressure in dogs. J Appl Physiol Respirat Environ Exercise Physiol 44:743-750, 1978

15. Cassidy SS, Eschenbacher WL, Johnson RLJ Jr: Reflex cardiovascular depression during unilateral lung hyperinflation in the dog. J Clin Invest 64:620-626, 1979

16. Tittley JG, Fremes SE, Weisel RD, et al: Hemodynamic and myocardial metabolic consequences of PEEP. Chest 88:496-502, 1985

17. Qvist J, Pontoppidan H, Wilson RS, et al: Hemodynamic responses to mechanical ventilation with PEEP: The effect of hypervolemia. Anesthesiology 42:45-55, 1975

18. Haynes JB, Carson SD, Whitney WP, et al: Positive endexpiratory pressure shifts left ventricular diastolic pressure-area curves. J Appl Physiol Respirat Environ Exercise Physiol 48:670676,1980 
19. Gabriele G, Rosenfeld CR, Fixler DE, et al: Continuous airway pressure breathing with the head-box in the newborn lamb: Effects on regional blood flows. Pediatrics 59:858-864, 1977

20. Maayan C, Eyal F, Mandelberg A, et al: Effect of mechanical ventilation and volume loading on left ventricular performance in premature infants with respiratory distress syndrome. Crit Care Med 14:858-860, 1986

21. Hausdorf G, Hellwege $\mathrm{HH}$ : Influence of positive and endexpiratory pressure on cardiac performance in premature infants: A Doppler-echocardiographic study. Crit Care Med 15:661-664, 1987

22. Dechert R, Wesley J, Schafer L, et al: Comparison of oxygen consumption, carbon dioxide production, and resting energy expenditure in premature and full-term infants. J Pediatr Surg 20:792798,1985

23. Powers SR, Mannal R, Neclerio $\mathbf{M}$, et al: Physiologic consequences of positive end-expiratory pressure (PEEP) ventilation. Ann Surg 178:265-272, 1973

24. Suter PM, Fairley HB, Isenberg MD: Optimum end- expiratory airway pressure in patients with acute pulmonary failure. N Engl J Med 292:284-289, 1975

25. Nelson LD: Continuous venous oximetry in surgical patients. Ann Surg 203:329-333, 1986

26. Davies GG, Mendenhall J, Symreng T: Measurement of right atrial oxygen saturation by fiberoptic oximetry accurately reflects mixed venous oxygen saturation in swine. J Clin Monit 4:99-102, 1988

27. Scalea TM, Holman M, Fuortes M, et al: Central venous blood oxygen saturation: An early, accurate measurement of volume during hemorrhage. J Trauma 28:725-732, 1988

28. Reinhart K, Rudolph T, Bredle OL, et al: Comparison of central-venous to mixed-venous oxygen saturation during changes in oxygen supply/demand. Chest 95:1216-1221, 1989

29. Martin GB, Carden DL, Nowak RM, et al: Central venous and mixed venous oxygen saturation: Comparison during canine open-chest cardiopulmonary resuscitation. Am J Emerg Med 3:495-497, 1985 